\title{
Expression of Chemokine CCL28 in Ulcerative Colitis Patients
}

\author{
Dong Seok Lee1, Kook Lae Lee ${ }^{1}$, Ji Bong Jeong ${ }^{1}$, Sue Shin², Su Hwan Kim¹, and Ji Won Kim¹ \\ Departments of ${ }^{1}$ Gastroenterology and ${ }^{2}$ Laboratory Medicine, SMG-SNU Boramae Medical Center, Seoul National University College \\ of Medicine, Seoul, Korea
}

\author{
Article Info \\ Received August 4, 2019 \\ Revised December 5, 2019 \\ Accepted December 6, 2019 \\ Published online February 28, 2020

Corresponding Author
Ji Won Kim
ORCID https://orcid.org/0000-0002-1214-5544
E-mail kjwjor@snu.ac.kr \\ Corresponding Author \\ ORCID https://orcid.org/0000-0002-1214-5544 \\ E-mail kjwjor@snu.ac.kr
}

Background/Aims: Ulcerative colitis (UC) is an inflammatory bowel disease for which new serological markers are required. The purpose of this study was to assess the role of the mucosaassociated epithelial chemokine CCL28 in UC.

Methods: The study included 50 patients; of these, 25 were patients with UC, and 25 were healthy controls. The levels of serum CCL28 were analyzed using enzyme-linked immunosorbent assay. CCL28 expression was analyzed by immunohistochemistry (IHC) in 15 representative colon tissues biopsied based on disease activity (UC patients with severe activity, five samples; UC patients with mild activity, five samples; healthy controls, five samples).

Results: The serum CCL28 levels were remarkably higher $(p<0.05)$ in patients with UC (median, $235.7 \mathrm{pg} / \mathrm{mL}$; IQR, 63.8 to $117.2 \mathrm{pg} / \mathrm{mL}$ ) than in healthy controls (median, 48.9, pg/mL; IQR, 35.9 to $42.0 \mathrm{pg} / \mathrm{mL}$ ). However, there was no significant difference in serum CCL28 according to disease extent or activity. In contrast, IHC analysis revealed a significant difference in CCL28 consistent with disease status, disease extent, and disease activity.

Conclusions: CCL28 could be useful for diagnosing UC. However, further validations of CCL28 on disease activity and severity are needed. (Gut Liver 2021;15:70-76)

Key Words: Colitis, ulcerative; CCL28; Serologic markers; Biomarker; Disease activity

\section{INTRODUCTION}

Various biological, chemical, and physical factors constantly bombard epithelial tissues lining the gastrointestinal (GI) tract. ${ }^{1}$ Immune cells that reside in epithelial tissue and circulating immune cells are collected to focal tissues in response to stimulation. ${ }^{1}$ The migration of leukocytes from the peripheral circulation to GI mucosa play an important role in immune surveillance. ${ }^{2-4}$ Endothelial cells interact with cytokine-activated leukocytes or other pro-inflammatory stimuli for rapid leukocyte migration through the endothelial barrier. ${ }^{5}$ Chemokine and integrins receptors on the leukocyte surface interact with cell-adhesion molecules and chemokines belonging to the endothelial and mucosal immunoglobulin superfamily, respectively, thereby accelerating the activation process. ${ }^{5}$

Chemokines are low-molecular-weight proteins that regulate the migration of circulating leukocytes to inflam- matory target position. Various types of cells release chemokines and more than 50 chemokines have been studied. Chemokines have been classified into four groups according to the functional and structural differences (CC, CXC, CX3C, and XCL1 chemokines). ${ }^{6,7}$ CCL28 is a member of the CC or $\beta$-chemokine subgroup. CCL28 and its chemokine receptors, CCR10 and CCR3, are involved in mucosal epithelial immunity. ${ }^{8,9}$ CCR10 and CCR3 are expressed by various subsets of lymphocytes and CCL28 is expressed by epithelial cells of various mucosal tissues. ${ }^{1,10}$ The chemoattraction of lymphocytes and the expression of chemokine receptors in response to chemokines play a significant role in barrier against infectious agents. ${ }^{11,12}$

Crohn's disease (CD) and ulcerative colitis (UC) and are the major kinds of chronic inflammatory bowel disease (IBD). The rapid infiltration and recruitment of leukocytes is the main feature of chronic inflammation in IBD. ${ }^{12,13}$ CCL28, which is mainly expressed in colonic vessels and 
epithelial cells, may play a crucial role in the mediating intercommunication of inflammatory cells with endothelial and epithelial cells and cause inflammation of the gut. Some current studies have present that the expression levels of serum CCL28 in rare inflammatory GI diseases varied according to disease activity. ${ }^{14-16}$ However, the expression levels of serum CCL28 in UC patients have not been studied.

This study compared the levels of CCL28 in the serum of UC patients and healthy control subjects. In addition, the expression levels of CCL28 in diseased and healthy colon tissue of UC patients and control subjects, respectively. Furthermore, the relationship between CCL28 levels and the extent and activity of the disease was examined.

\section{MATERIALS AND METHODS}

\section{Study cohort and design}

Total 50 patients were registered in this study from January 2014 to December 2014. Of these, 25 were patients with UC, who were diagnosed and monitored at the SMGSNU Boramae Medical Center. The other 25 were healthy control subjects, who had a colonoscopy at the SMG-SNU Boramae Medical Center during routine checkup. The results of clinical, endoscopic and histopathological analyses were used to diagnose UC. ${ }^{17}$ The disease activity of UC was determined at the time of participation in this study and grouped as mild, moderate, and severe activity according to the Mayo score index as described by Rutgeerts et al. ${ }^{18}$ Patients with a total Mayo score of 11 to 12 points (the score can scope from 0 to 12 with higher scores, being indicative of more severe disease activity) were classified as having severe activity, while patients with a total Mayo score of 3 to 5 points were classified as having mild activity. Clinical characteristics of the 50 patients have been described in Table 1.

Routine laboratory investigations used to diagnose UC, included hemoglobin and hematocrit levels, platelet count, red and white blood cell count, albumin, total protein, cholesterol, triglycerides, and high sensitivity C-reactive protein. The high sensitivity C-reactive protein was used as a marker of inflammation, and levels of $0.5 \mathrm{mg} / \mathrm{dL}$ or less were considered normal values. Patients with any chronic systemic disorder, including malignant hypertension, coronary heart disease, uncontrolled diabetes mellitus, adrenal insufficiency, hypo- or hyperthyroidism, or autoimmune disease or any malignancy, were excluded from the study. This study included people who had no other bowel disease other than UC to rule out these other possibilities. In addition, bacterial and viral tests were performed when blood and colon tissues were examined to rule out an increase in CCL28 levels brought about by infectious diseases. Bacteria and viruses were not detected in the blood and colon tissues.

The research protocol was approved by the Institutional

Table 1. Patient Characteristics

\begin{tabular}{|c|c|c|c|}
\hline Variable & UC patient $(n=25)$ & Control (n=25) & p-value \\
\hline Age, yr & $46.22 \pm 12.92$ & $49.83 \pm 7.79$ & 0.133 \\
\hline Sex, male/female & $13 / 12$ & $13 / 12$ & 1.000 \\
\hline \multicolumn{4}{|l|}{ Comorbidity } \\
\hline Hypertension & 0 & 0 & 1.000 \\
\hline Diabetes & 0 & 0 & 1.000 \\
\hline Other disease & 0 & 0 & 1.000 \\
\hline Current smoker & $2(8.0)$ & $2(8.0)$ & 1.000 \\
\hline C-reactive protein, $\mathrm{mg} / \mathrm{dL}$ & $0.93 \pm 1.64$ & $0.02 \pm 0.04$ & 0.007 \\
\hline Extent of disease & & & - \\
\hline Pancolitis & $17(68.0)$ & & \\
\hline Left-sided colitis & $4(16.0)$ & & \\
\hline Proctitis & $4(16.0)$ & & \\
\hline Mayo score & $7.08 \pm 3.23$ & & - \\
\hline Mild (n=10) & $3.80 \pm 1.32$ & & \\
\hline Moderate $(n=10)$ & $8.30 \pm 1.70$ & & \\
\hline Severe $(n=5)$ & $11.20 \pm 0.45$ & & \\
\hline Medications & & & - \\
\hline 5-Aminosalicylates or sulfasalazine & $25(100)$ & & \\
\hline Corticosteroids & 8 (32.0) & & \\
\hline Azathioprine & $11(44.0)$ & & \\
\hline Biological agents & $1(4.0)$ & & \\
\hline
\end{tabular}

Data are presented as mean \pm SD or number $(\%)$.

UC, ulcerative colitis. 
Review Board at SMG-SNU Boramae Medical Center (IRB number: 26-2014-55), and all patients provided written informed consent for the use of their personal information in manuscripts.

\section{Assessment of serum CCL28 concentration by enzyme-linked immunosorbent assay}

The levels of serum CCL28 were analyzed using a commercial CCL28 human enzyme-linked immunosorbent assay kit (Abcam, Cambridge, UK) as described by Yoon et al. ${ }^{19}$ The serum samples were thawed and vortexed before analysis of the enzyme-linked immunosorbent assay. Centrifuged supernatant was used for CCL28 measurements based on the manufacturer's instructions. Stop solution was added to each well and a spectrophotometric plate-reader was used to measure the absorption values (Microplate Reader; BioTek Instruments Inc., Winooski, VT, USA). All samples were evaluated repeatedly.

\section{Detection of CCL28 by immunohistochemistry analysis and scoring}

Pathological mucosal lesions of UC patients and normal mucosa of healthy control subjects were biopsied during colonoscopic examinations. For immunohistochemistry (IHC) analysis, 15 representative samples of mucosa were analyzed, five samples were from UC patients with severe disease activity, five samples were from UC patients with mild disease activity, and five samples were from healthy control subjects. Protein levels of CCL28 were detected using IHC. Scoring for staining was conducted semiquantitatively based on the staining intensity and percentage staining of CCL28 protein. The intensity of staining was graded as follows: 3 , strong; 2 , moderate; 1 , low; and 0 , no staining. The percentages of stained cells were scored as follows: 3 points, $>75 \%$; 2 points, $20 \%$ to $75 \%$; 1 point, $5 \%$ to $19 \%$; 0 point $<5 \%$ of cells stained. ${ }^{20}$ The total score was the multiplication product of the scores for the staining intensity and percentage. In this study, the final total scores of $<3$ and $\geq 3$ with respect to CCL28 expression were classified as low and high expression, respectively.

\section{Statistical analysis}

Continuous variables were described as mean \pm standard deviation and analyzed using the Mann-Whitney U-test or Student t-test. Categorical variables were compared using the Fisher exact test or chi-square test. CCL28 levels were compared using the Kruskal-Wallis test or an analysis of variance according to the extent or activity of disease. Analyses were conducted by the statistical software package SPSS 19.0 (SPSS Inc., Chicago, IL, USA). p-values of $<0.05$ were considered statistically significant.

\section{RESULTS}

\section{Patient characteristics}

Table 1 shows the clinical characteristics of the 50 test subjects. There were no outstanding differences between the 25 UC patients and 25 healthy control subjects with respect to age and gender $(p>0.05)$. The mean ages \pm standard deviations of UC patients and healthy control subjects were $45.1 \pm 13.9$ and $50.0 \pm 7.8$, respectively. The extent of disease in UC patients was categorized as non-pancolitis $(n=8)$ or pancolitis $(n=17)$. Of the 25 diagnosed UC patients, five were determined as showing severe disease activity, 10 were determined as showing moderate disease activity, and 10 were determined as showing mild activity.

\section{Serum CCL28 concentrations in UC patients and healthy control subjects}

The serum CCL28 levels of UC patients and healthy control subjects are shown in Fig. 1. The serum CCL28 levels were remarkably higher in the UC patients $(\mathrm{p}<0.05$; median, $235.7 \mathrm{pg} / \mathrm{mL}$; interquartile range [IQR], 63.8 to $117.2 \mathrm{pg} / \mathrm{mL}$ ) than in healthy control subjects (median, $48.9 \mathrm{pg} / \mathrm{mL}$; IQR, 35.9 to $42.0 \mathrm{pg} / \mathrm{mL}$ ).

\section{Serum CCL28 concentrations in UC patients according to the disease extent}

The mean serum CCL28 concentrations were higher in patients with pancolitis ( $187.5 \mathrm{pg} / \mathrm{mL}$ IQR, 61.8 to 95.5 $\mathrm{pg} / \mathrm{mL})$ than those with non-pancolitis $(150 \mathrm{pg} / \mathrm{mL}$; IQR, 74.4 to $151.8 \mathrm{pg} / \mathrm{mL})(\mathrm{p}=0.215)$ (Fig. 2). However, the concentration of serum CCL28 did not show a statistically significant difference with respect to the extent of disease $(\mathrm{p}=0.262)$.

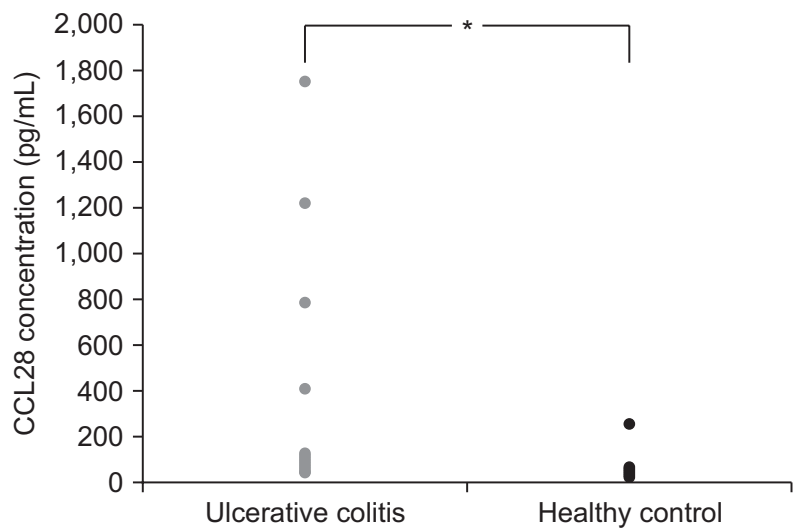

Fig. 1. Serum CCL28 levels in patients with ulcerative colitis ( $n=25$ ) and healthy controls ( $n=25$ ). ${ }^{*} p<0.05$, versus controls. 


\section{Serum CCL28 concentrations in UC patients according to the disease activity}

The mean serum CCL28 concentrations were higher in patients with severe activity $(251.1 \mathrm{pg} / \mathrm{mL}$; IQR, 61.1 to $126.4 \mathrm{pg} / \mathrm{mL})$ than in those with mild activity $(181.2$ $\mathrm{pg} / \mathrm{mL}$; IQR, 62.6 to $241 \mathrm{pg} / \mathrm{mL}$ ) (Fig. 3). However, serum CCL28 concentrations did not show a statistically significant difference with respect to the disease activity $(\mathrm{p}=0.885)$.

\section{Protein levels of CCL28 in colon tissue of UC patients and healthy controls}

To investigate the expression of CCL28 in colon tissues, biopsy samples were obtained from patients with UC and healthy control subjects. IHC analysis revealed increased expression of CCL28 in actively diseased tissues of UC patients compared to those in healthy controls. The mean scores calculated for IHC analysis were $3 \pm 0$ (high CCL28 expression) for the samples from UC patients with severe disease activity ( $\mathrm{n}=5), 0.80 \pm 0.84$ (low CCL28 expression) for samples from UC patients with mild disease activity

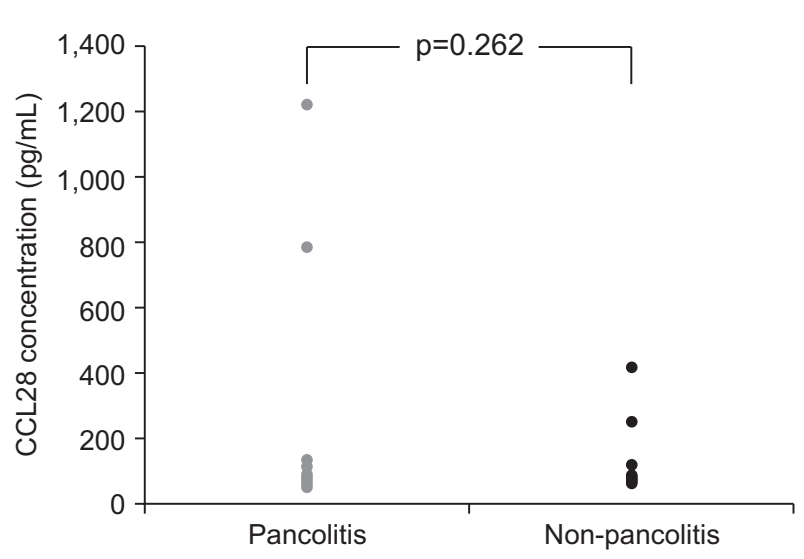

Fig. 2. Serum CCL28 levels in ulcerative colitis patients with $(n=17)$ and without $(\mathrm{n}=8)$ pancolitis. $(\mathrm{n}=5)$, and $0 \pm 0$ (no protein detected) for healthy controls $(\mathrm{n}=5)$. Representative IHC images of CCL28 expression according to disease status of UC are shown in Fig. 4.

\section{The sensitivity and specificity of CCL28 for the diagnosis of UC}

Fig. 5 presents the receiver operating characteristic curve analysis of serum CCL28 for the diagnosis of UC. The sensitivity and specificity were $96 \%$ and $92 \%$, respectively.

\section{DISCUSSION}

Although anti-Saccharomyces cerevisiae antibodies and anti-neutrophil cytoplasmic autoantibodies (pANCA) are highly sensitive in the diagnosis of $\mathrm{UC}$ and $\mathrm{CD}$ respectively, other serologic markers are not useful in the diagnosis of IBD because of their limited sensitivity. ${ }^{21}$ In other GI tract disorders like celiac disease, the serologic marker anti-Saccharomyces cerevisiae antibodies, as well as auto-

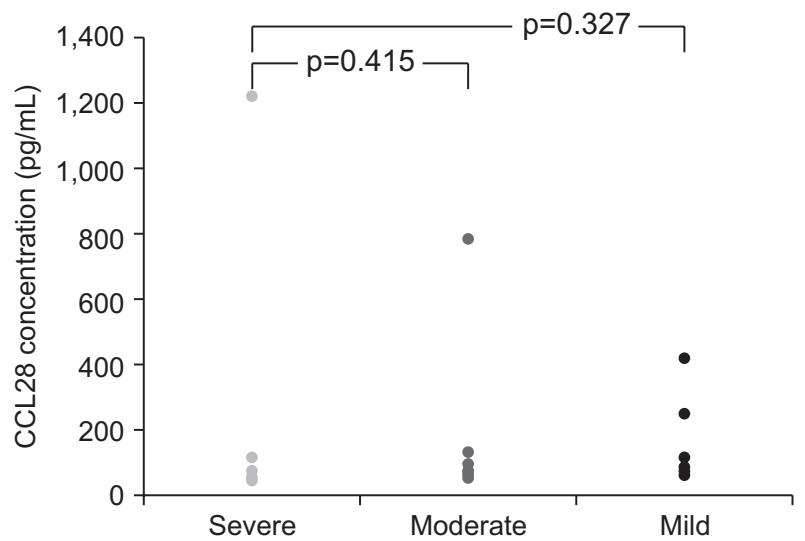

Fig. 3. Serum CCL28 levels in ulcerative colitis patients with severe disease activity $(n=5)$, moderate disease activity $(n=10)$, and mild disease activity $(n=10)$.
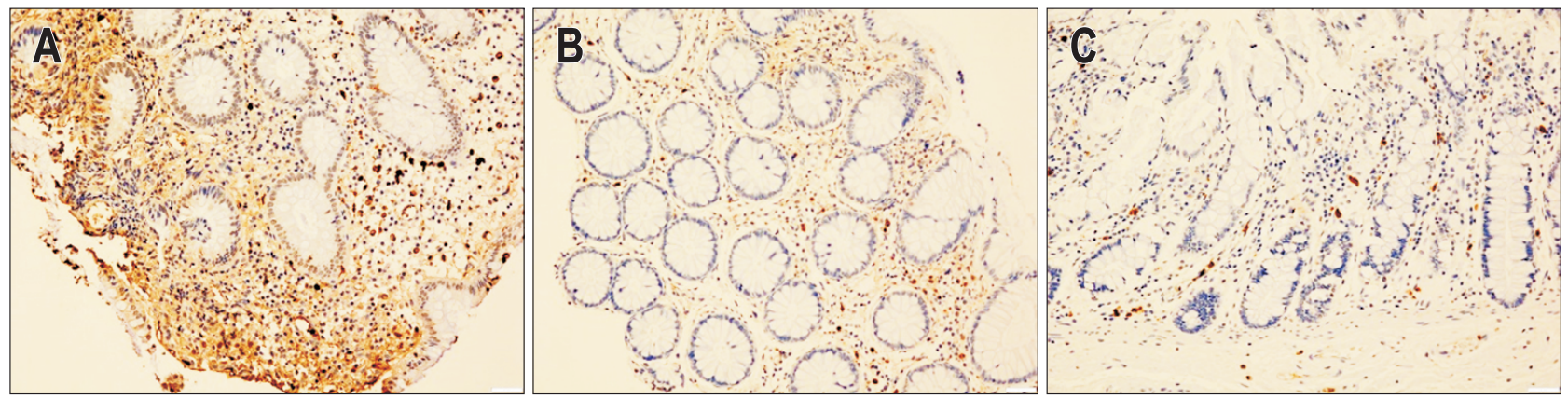

Fig. 4. Representative images of immunohistochemistry (IHC) analysis of CCL28 in mucosal tissue extracted from colon biopsy samples. (A) Sample from an ulcerative colitis (UC) patient with severe disease activity. The mean IHC score $\pm S D$ of all samples from this group $(n=5)$ was $3 \pm 0(\times 100)$. (B) Sample from a UC patient with mild disease activity. The mean IHC score $\pm S D$ of all samples from this group $(n=5)$ was $0.80 \pm 0.84(\times 100)$. (C) Sample from a normal tissue obtained from a healthy control. The mean IHC score $\pm S D$ of all samples from this group $(n=5)$ was $0 \pm 0(\times 100)$. 


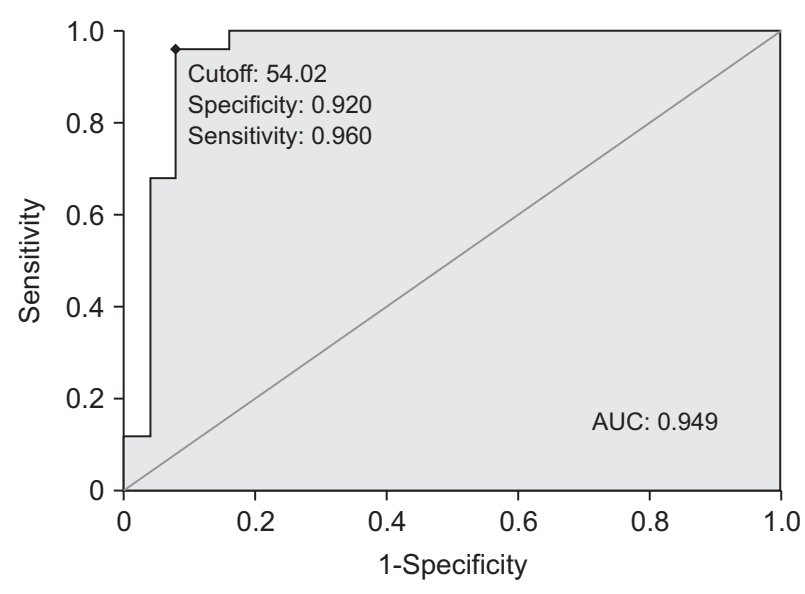

Fig. 5. Receiver operating characteristic curve analysis of serum CCL28 for the diagnosis of ulcerative colitis. The sensitivity was $96 \%$ and specificity was $92 \%$. The gray line is the reference line and the black line is the receiver operating characteristic (ROC) curve.

AUC, area under the ROC curve.

antibodies against exocrine pancreas are increased..$^{22}$ Increasing the panel of antibodies for the diagnosis of IBD is necessary to improve the rates of diagnosis.

Serologic markers can also have prognostic value in stratifying disease phenotypes. Stratification of the IBD subtype by disease progression and complications helps early determination of appropriate treatment modalities and improves disease outcome. There are correlational studies between anti-glycan antibodies (anti-chitosan, antimannobioside, anti-laminaribioside, anti-laminarin, antichitin antibodies, and anti-Saccharomyces cerevisiae antibodies) and more complicated CD phenotypes, and higher frequency of CD-related abdominal surgery. ${ }^{23-26}$ This association is proportional to the serological response (multiple antibodies and higher titers). However, apart from pAN$\mathrm{CA}$, there are no other known serologic markers for UC. Furthermore, the association of pANCA with UC disease phenotype is controversial. In a study of 366 IBD patients, pANCA was not associated with disease activity, location, disease duration, disease extent, medical treatment, or previous bowel surgery in UC patients. ${ }^{27}$ In other UC cohort studies, pANCA was extremely associated with more severe disease course. ${ }^{28-30}$ These observations highlight the significance of the results of this study, which indicate that chemokine CCL28 may be used as a new serologic marker of UC.

Serologic markers are also expected to be useful in monitoring disease activity and response to drug therapy. Serologic markers are likely to help establish a personalized treatment strategy. However, according to the available data, serial measurements of IBD serological markers have not been used in monitoring disease activity. ${ }^{31-33}$
A previous study investigated cellular CCL28 mRNA levels in vivo in human colon and small intestinal xenografts, as well as in vitro, following stimulation with interleukin- $1 \alpha$, interleukin- 6 , and tumor necrosis factor- $\alpha$ or interferon- $\gamma \cdot{ }^{34}$ A pilot study of 11 patients with IBD, including UC and CD, was published in 2017. ${ }^{16}$ However, this pilot study mentioned serum CCL28 as one among the several chemokine studied and the number of patients was too small.

This paper is the first report and to examine the association between UC patients and CCL28. This study compared the CCL28 levels in serum and colon tissues of UC patients and healthy control subjects, and assessed the relationship between CCL28 levels and the extent and activity of the disease. Serum CCL28 levels were markedly higher in patients with UC than in healthy control subjects. However, no statistical difference was observed in the concentration of serum CCL28 with respect to the extent of disease or activity. These results appear to be due to study limitations such as the lack of patient numbers and disease state. The number of UC patients with severe state disease was relatively small (20\%), and the C-reactive protein levels in UC patients were mostly in the normal range. In addition, the levels of serum CCL28 were likely reduced by the drugs used by UC patients. ${ }^{35}$ Therefore, it is likely that the measured CCL28 in most UC patients reflected its reduced levels in the serum. ${ }^{35}$ To compensate for this drawback, we used biopsy samples retaining the high CCL28 levels for a long time. In IHC analysis, the level of CCL28 was proportional to disease activity.

For the diagnosis of UC, endoscopic findings are more important than CCL28 serum concentrations. Elevated serum CCL28 concentrations cannot be used as a single diagnostic tool for UC because CCL28 levels can increased in various enteritis-related diseases $(\mathrm{CD}$, infectious colitis, etc.). ${ }^{15,34,36}$ However, if clinical characteristics and endoscopic findings correspond to UC, elevated CCL28 levels may be helpful for the diagnosis of UC.

This study has several limitations. First, the patients were enrolled retrospectively. Therefore, the levels of serum CCL28 were not measured at initial diagnosis and disease courses, such as steroid dependency, use of immunosuppressive agents, or operative therapy, were not evaluated. Second, because of the small number of patients, statistically significant differences in serum CCL28 concentrations of UC patients with varying disease activity were not observed. Large-scale research is necessary to clear up the interrelation between CCL28 and the extent and activity of disease. Finally, the CCL28 levels were only measured in UC patients and not in CD patients. A study on CCL28 levels in $\mathrm{CD}$, as the second main type of IBDs, is needed. 
In conclusion, this study showed increased CCL28 levels in colon tissue and serum of UC patients as compared to those in healthy controls. We believe that CCL28 could serve as a useful supplementary diagnostic tool for diagnosing UC. However, of the effect of CCL28 on disease activity and severity need to be validated further.

\section{CONFLICTS OF INTEREST}

No potential conflict of interest relevant to this article was reported.

\section{AUTHOR CONTRIBUTIONS}

Concept: D.S.L., J.W.K. Design: D.S.L., J.W.K. Supervision: K.L.L., J.W.K. Resource: S.S., J.B.J. Materials: S.S., J.B.J., S.H.K. Data collection and/or processing: D.S.L., J.W.K., J.B.J., S.H.K. Analysis and/or interpretation: D.S.L., J.W.K. Literature search: D.S.L., J.W.K., S.H.K. Writing manuscript: D.S.L., J.W.K. Critical reviews: K.L.L., J.W.K.

\section{ORCID}

Dong Seok Lee https://orcid.org/0000-0003-2231-0563

Kook Lae Lee https://orcid.org/0000-0001-6676-9451

Ji Bong Jeong https://orcid.org/0000-0003-4553-1721

Sue Shin https://orcid.org/0000-0003-4791-8671

Su Hwan Kim https://orcid.org/0000-0001-6444-7969

Ji Won Kim https://orcid.org/0000-0002-1214-5544

\section{REFERENCES}

1. Xiong N, Fu Y, Hu S, Xia M, Yang J. CCR10 and its ligands in regulation of epithelial immunity and diseases. Protein Cell 2012;3:571-580.

2. Delves PJ, Roitt IM. The immune system: first of two parts. N Engl J Med 2000;343:37-49.

3. Delves PJ, Roitt IM. The immune system: second of two parts. N Engl J Med 2000;343:108-117.

4. McIntyre TM, Prescott SM, Weyrich AS, Zimmerman GA. Cell-cell interactions: leukocyte-endothelial interactions. Curr Opin Hematol 2003;10:150-158.

5. Charo IF, Ransohoff RM. The many roles of chemokines and chemokine receptors in inflammation. N Engl J Med 2006;354:610-621.

6. Cyster JG. Chemokines, sphingosine-1-phosphate, and cell migration in secondary lymphoid organs. Annu Rev Immu- nol 2005;23:127-159.

7. Gerard C, Rollins BJ. Chemokines and disease. Nat Immunol 2001;2:108-115.

8. Eksteen B, Miles A, Curbishley SM, et al. Epithelial inflammation is associated with CCL28 production and the recruitment of regulatory $\mathrm{T}$ cells expressing CCR10. J Immunol 2006;177:593-603.

9. Mohan T, Deng L, Wang BZ. CCL28 chemokine: an anchoring point bridging innate and adaptive immunity. Int Immunopharmacol 2017;51:165-170.

10. Kim BG, Kim JW, Jeong JB, et al. Expression of human $T$ cell-activating CXC chemokines in inflammatory bowel disease. Intest Res 2004;2:58-64.

11. Mora JR. Homing imprinting and immunomodulation in the gut: role of dendritic cells and retinoids. Inflamm Bowel Dis 2008; 14:275-289.

12. von Andrian UH, Mackay CR. T-cell function and migration. Two sides of the same coin. N Engl J Med 2000;343:1020-1034.

13. Baumgart DC, Carding SR. Inflammatory bowel disease: cause and immunobiology. Lancet 2007;369:1627-1640.

14. Matsuo K, Nagakubo D, Yamamoto S, et al. CCL28-deficient mice have reduced IgA antibody-secreting cells and an altered microbiota in the colon. J Immunol 2018;200:800-809.

15. Rashidiani S, Jalili A, Babaei E, et al. The chemokine CCL28 is elevated in the serum of patients with celiac disease and decreased after treatment. Am J Clin Exp Immunol 2017;6:60-65.

16. Zwicker S, Lira-Junior R, Höög C, Almer S, Boström EA. Systemic chemokine levels with "gut-specific" vedolizumab in patients with inflammatory bowel disease-a pilot study. Int J Mol Sci 2017;18:1827.

17. Choi CH, Jung SA, Lee BI, et al. Diagnostic guideline of ulcerative colitis. Korean J Gastroenterol 2009;53:145-160.

18. Rutgeerts P, Sandborn WJ, Feagan BG, et al. Infliximab for induction and maintenance therapy for ulcerative colitis. $\mathrm{N}$ Engl J Med 2005;353:2462-2476.

19. Yoon JH, Oh S, Shin S, et al. Plasma CC-chemokine ligand 28 level is correlated with hematopoietic stem cells in human cord blood. Transfusion 2015;55:1008-1012.

20. Ohuchida K, Mizumoto K, Ishikawa N, et al. The role of S100A6 in pancreatic cancer development and its clinical implication as a diagnostic marker and therapeutic target. Clin Cancer Res 2005;11:7785-7793.

21. Kuna AT. Serological markers of inflammatory bowel disease. Biochem Med (Zagreb) 2013;23:28-42.

22. Barta Z, Csípõ I, Szabó GG, Szegedi G. Seroreactivity against Saccharomyces cerevisiae in patients with Crohn's disease and celiac disease. World J Gastroenterol 2003;9:2308-2312.

23. Rieder F, Schleder S, Wolf A, et al. Serum anti-glycan antibodies predict complicated Crohn's disease behavior: a co- 
hort study. Inflamm Bowel Dis 2010;16:1367-1375.

24. Rieder F, Schleder S, Wolf A, et al. Association of the novel serologic anti-glycan antibodies anti-laminarin and antichitin with complicated Crohn's disease behavior. Inflamm Bowel Dis 2010;16:263-274.

25. Simondi D, Mengozzi G, Betteto S, et al. Antiglycan antibodies as serological markers in the differential diagnosis of inflammatory bowel disease. Inflamm Bowel Dis 2008;14:645651.

26. Ferrante M, Henckaerts L, Joossens M, et al. New serological markers in inflammatory bowel disease are associated with complicated disease behaviour. Gut 2007;56:1394-1403.

27. Oudkerk Pool M, Ellerbroek PM, Ridwan BU, et al. Serum antineutrophil cytoplasmic autoantibodies in inflammatory bowel disease are mainly associated with ulcerative colitis: a correlation study between perinuclear antineutrophil cytoplasmic autoantibodies and clinical parameters, medical, and surgical treatment. Gut 1993;34:46-50.

28. Høie O, Aamodt G, Vermeire S, et al. Serological markers are associated with disease course in ulcerative colitis: a study in an unselected population-based cohort followed for 10 years. J Crohns Colitis 2008;2:114-122.

29. Vecchi M, Bianchi MB, Sinico RA, et al. Antibodies to neutrophil cytoplasm in Italian patients with ulcerative colitis: sensitivity, specificity and recognition of putative antigens. Digestion 1994;55:34-39.
30. Sandborn WJ, Landers CJ, Tremaine WJ, Targan SR. Association of antineutrophil cytoplasmic antibodies with resistance to treatment of left-sided ulcerative colitis: results of a pilot study. Mayo Clin Proc 1996;71:431-436.

31. Prideaux L, De Cruz P, Ng SC, Kamm MA. Serological antibodies in inflammatory bowel disease: a systematic review. Inflamm Bowel Dis 2012;18:1340-1355.

32. Bossuyt X. Serologic markers in inflammatory bowel disease. Clin Chem 2006;52:171-181.

33. Reumaux D, Colombel JF, Masy E, et al. Anti-neutrophil cytoplasmic auto-antibodies (ANCA) in ulcerative colitis (UC): no relationship with disease activity. Inflamm Bowel Dis 2000;6:270-274.

34. Ogawa H, Iimura M, Eckmann L, Kagnoff MF. Regulated production of the chemokine CCL28 in human colon epithelium. Am J Physiol Gastrointest Liver Physiol 2004;287:G1062-G1069.

35. Aziz N, Detels R, Quint JJ, Li Q, Gjertson D, Butch AW. Stability of cytokines, chemokines and soluble activation markers in unprocessed blood stored under different conditions. Cytokine 2016;84:17-24.

36. Hansson M, Hermansson M, Svensson H, et al. CCL28 is increased in human Helicobacter pylori-induced gastritis and mediates recruitment of gastric immunoglobulin Asecreting cells. Infect Immun 2008;76:3304-3311. 